\section{Phyto- und Mykotoxine (3)}

\section{Lektine}

Jean-Michel Jeannin

\section{Einleitung}

Die Geschichte der Lektine beginnt mit dem Jahr 1888, als Peter Hermann Stillmark seine Entdeckung des Hämagglutinins (ein Lektin) im Rahmen seines Doktorats vorstellte. Das Hämagglutinin (Abb. 1) hatte er aus dem Wunderbaum (Ricininus communis) isoliert. Die Substanz galt als hochtoxisch [1]. Lektine sind sekundäre Pflanzenstoffe. Biochemisch handelt es sich um Proteine oder Glykoproteine. Lektine kommen in verschiedenen Pflanzenarten, in Bakterien, in Pilzen und in Säugetieren vor. Eine Besonderheit der Lektine ist ihre Verwendung in der Forschung [2]. Entsprechend der Vielfalt und Ausdehnung des Themas kann der vorliegende Beitrag nur einzelne ausgewählte Aspekte behandeln und muss sich auf Lektine von Pflanzen beschränken (Tab. 1).

\section{Biochemie und Toxikologie ausgewählter Lektine}

Lektine sind komplexe Proteine. Sie bestehen aus zwei Ketten, einem Effektomer (A-Kette) und einem Haptomer
(B-Kette). Lektine sind stabile Moleküle und widerstehen einem Abbau durch Proteasen. Hitze über $65^{\circ} \mathrm{C}$ zerstört die Lektine [3], wobei angeraten wird, Bohnen bei $100^{\circ} \mathrm{C}$ zu kochen, damit alle Toxine zerstört werden [4]. Die pflanzlichen Lektine sind meistens in den Samen loka-

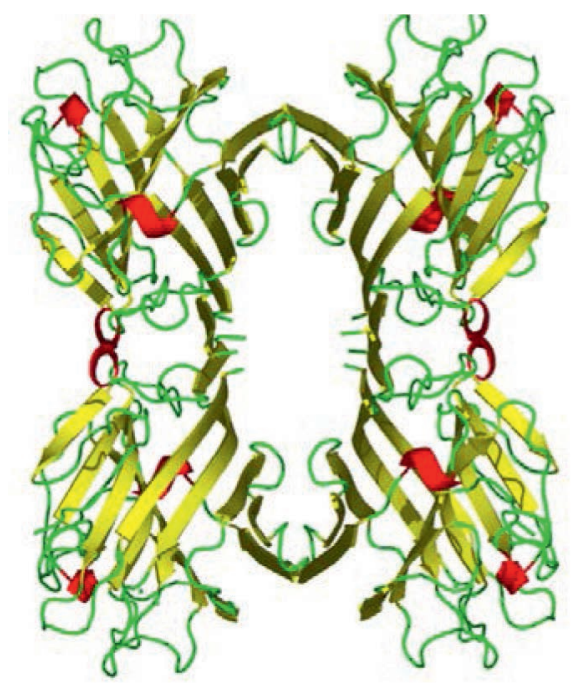

Abb. 1.

Hämagglutinin (Quelle: Wikipedia).

Tab. 1. Lektinhaltige Pflanzenarten (modifiziert nach $[2,3]$ )

\begin{tabular}{|c|c|c|}
\hline Botanischer Name & Umgangssprachliche Bezeichnung & Lektin \\
\hline Abrus precatorius & Paternostererbse & Abrin A-d \\
\hline Arachis hypogaea & Erdnuss & Erdnussagglutinin \\
\hline Canavalia ensiformis & Jackbohne & Concanavalin A \\
\hline Caragana arborescens & Gewöhnlicher Erbsenstrauch & 1-Canavanin \\
\hline Galanthus nivalis & Kleines Schneeglöckchen & Schneeglöckchen-Lektin (GNA) \\
\hline Glycine $\max (\mathrm{L}$.$) Merr.$ & Sojabohne & Soyabohnen-Lektin (SBA) \\
\hline Jatropha curcas & Purgiernussbaum & Curcin \\
\hline Lens culinaris & Linse & Linsen-Lektin (LCH) \\
\hline Musa acuminata & Banane & Bananen-Lektin/BanLec) \\
\hline Phaseolus vulgaris & Gartenbohne (unter anderem Rote Kidneybohne) & Phasine (unter anderem Phaseolin), Phytohämagglutinin \\
\hline Phytolacca americana & Amerikanische Kermesbeere & verschiedene \\
\hline Ricinus communis & Rizinusstrauch, Wunderbaum & Ricin \\
\hline Robinia pseudoacacia & Robinie, Gewöhnliche Scheinakazie & Robin \\
\hline Sambucus nigrus & Schwarzer Holunder & Sambunigrin \\
\hline Triticum L. & Weizen & Weizenkeim-Lektin (WGA) \\
\hline Ulex europaeus & Stechginster & Stechginster-Lektine \\
\hline Vicia faba & Ackerbohne & verschiedene \\
\hline Vicia villosa & Wicke & Wicken-Lektin (VVL) \\
\hline Viscum album & Mistel & Mistellektine I-III (MI-III) \\
\hline Wisteria sinensis & Blauregen, Glyzine & hämagglutinierende Lektine \\
\hline
\end{tabular}

\section{KARGER}

Fax +497614520714 Information@Karger.com www.karger.com 
lisiert. Lektine binden sich mit dem Haptomer an Zuckerstrukturen der Zelloberfläche, wo sie durch Endozytose in die Zelle aufgenommen werden. In der Zelle blockieren sie die ribosomale Proteintranslation; die Zelle bildet in der Folge kein Protein mehr und stirbt ab [3]. Phytohämagglutinine binden an Erythrozyten und bringen sie so zum Agglutinieren [4].

Ricin aus dem Wunderbaum (Abb. 2) ist ein Gemisch von vier Lektinen (RCL $1-4)$. Die $\mathrm{LD}_{50}$ beträgt bei der Maus $0,1 \mu \mathrm{g} / \mathrm{kg}$ intraperitoneal; die letale orale Dosis für den Menschen beträgt $1 \mathrm{mg} / \mathrm{kg}$. Für Kinder sind 5-6 Rizinussamen tödlich, für Erwachsene 20.

Die $\mathrm{LD}_{50}$ des Abrins aus der Paternostererbse beträgt bei der Maus $40 \mu \mathrm{g} / \mathrm{kg}$ intraperitoneal, $0,7 \mu \mathrm{g} / \mathrm{kg}$ i.v. und $2 \mathrm{mg} / \mathrm{kg}$ oral sowie $0,06 \mu \mathrm{g} / \mathrm{kg}$ i.v. beim Kaninchen. 2 Abrus-Samen (ca. 0,5 g) können für den Menschen tödlich sein, während ein einziger Samen bei Kindern tödlich ist. $60 \mathrm{~g}$ der Samen sind für ein Pferd tödlich [3].

Von den Hülsenfrüchten hat die rohe Rote Kidneybohne (Abb. 3) mit 20 000-70 000 hämagglutinierenden Einheiten (HAU) den höchsten Gehalt an Phytohämagglutininen, gefolgt von der rohen Weissen Kidneybohne mit 7000-23 000 HAU und der Gartenbohne mit 10007000 HAU. Die gekochten Bohnen sind unschädlich [4].

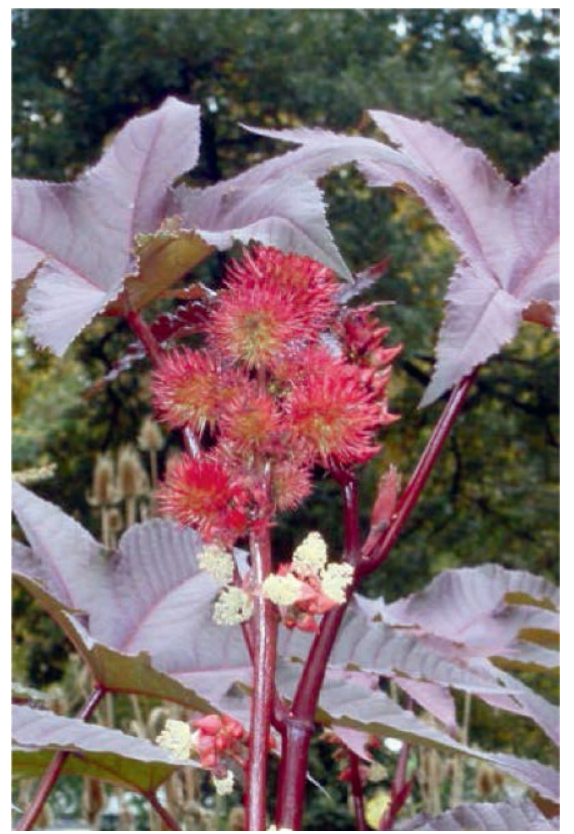

Abb. 2.

Ricinus communis (Rizinusstrauch). Botanischer Garten Basel, 2012.

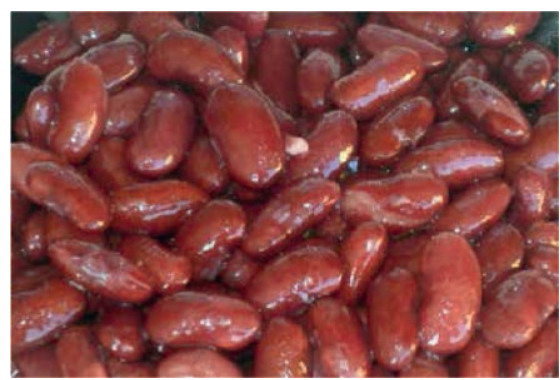

Abb. 3.

Phaseolus vulgaris (Rote Gartenbohne, Kidneybohne).
Tab. 2. Giftklassen der WHO (nach [3])

\begin{tabular}{lll}
\hline Klasse & Bezeichnung & $\mathrm{LD}_{50}$ Ratte, $\mathrm{mg} / \mathrm{kg} \mathrm{KG}$ \\
\hline Ia & äusserst giftig & $\leq 5$ \\
Ib & sehr giftig & $5-50$ \\
II & giftig & $50-500$ \\
III & schwach giftig & $>500$ \\
\hline
\end{tabular}

Abroin, Phasin und Ricin gehören zur Giftklasse Ia (Tab. 2). Der Mechanismus der Vergiftung ist nicht bekannt. Eine intramuskuläre Anwendung erhöht die Toxizität um ein Vielfaches [3].

Die Mistel (Viscum album) enthält die Mistel-Lektine I-III (MI-III) sowie ein Chitin-bindendes Lektin. MI bindet an D-Galactosereste, MII und MII an N-Acetylgalactosaminreste. Das Chitin-bindende Lektin bindet an N-Acetyl-D-Glucosaminreste. Ein in Escherichia coli hergestelltes rekombinantes Lektin weist Spezifitäten sowohl für D-Galactose- als auch für N-Acetylgalactosaminreste auf [5].

\section{Vergiftung und Notfallmassnahmen}

Die Symptome einer Vergiftung bestehen aus Übelkeit, Erbrechen, schwerer Magen-Darm-Entzündung, blutigem Durchfall, Herzrasen (Tachykardie) und grossem Flüssigkeitsverlust. Schwere Vergiftungen rufen Herz-, Nieren- und Pankreasversagen hervor; der Tod tritt infolge Atemlähmung oder Urämie 3-4 Tage nach der Einnahme ein. Kontakt mit der Haut oder mit der Lunge löst allergische Reaktionen bis zum anaphylaktischen Schock aus. Notfallmassnahmen sind sofortiges Auslösen von Erbrechen, Verabreichung von Natriumsulfat und Medizinalkohle sowie Einweisung in ein Spital. Spezifische Antidote sind nicht bekannt.

\section{Verwendung}

\section{Erforschung von Zelloberflächen}

Lektine binden mit hoher Spezifität an ihre Zielstruktur. Sie eignen sich deswegen zur Erforschung von zuckerhaltigen Strukturen auf Zelloberflächen. Verschiedene Lektine vermögen in vitro Lymphozyten zur Mitose anzuregen (Phytohämagglutinin, Concanavalin A). Diese Entdeckung führte ihrerseits zur Entdeckung von Interleukin-2 durch Robert C. Gallo und sein Team [1].

\section{Behandlung von Malignomen}

Das Weizenkeim-Lektin (WGA), das Concanavalin A und das Soyabohnen-Lektin haben die Fähigkeit, bevorzugt maligne Zellen zu agglutinieren, was interessante Perspektiven für die Behandlung von Malignomen bietet. Die Beobachtung lieferte eine gewisse Evidenz dafür, dass 
Veränderungen der Zelloberflächen-Zucker mit der Entstehung von Krebs verbunden sind [4]. In einer Insitu-Modellstudie konnte mit Concanavalin A ein malignes murines Hepatom eradiziert werden. Die Autoren nehmen an, dass Concanavalin A durch die Aufnahme in die Zelle die Autophagie, d.h. ihre Selbstzerstörung, auslöst und so den Tumor zerstört. Gleichzeitig würde die Mitose der Lymphozyten stimuliert [6]. Die Behandlung von Karzinomen mit einem Mistelpräparat wird kontrovers diskutiert [5], wenn auch die Eigenschaften der darin enthaltenen Lektine eine solche Behandlung nach Ansicht des Autors plausibel erscheinen lässt.

\section{Antivirale Wirkungen}

Ein Lektin aus Bananen (Abb. 4) erwies sich als hochwirksamer Hemmer der HIV-Replikation. Nach Ansicht der Autoren bietet sich hier die Möglichkeit, die Übertragung des HIV-1 zu verhindern [7].

\section{Blutgruppen-Identifikation}

Die Identifikation von Blutgruppen ist die älteste Anwendung von Lektinen. Hierbei wird der Umstand genutzt, dass ein Lektin-Molekül an die Zielstruktur mehrerer Erythrozyten bindet, was zur im Testsystem gut sichtbaren Agglutination führt. Die Spezifität wird

\section{Literatur}

1 Sharon N, et al: History of lectins: from hemagglutinins to biological recognition molecules. Glycobiology 2004;14:53R-62R.

2 Lektine. http://de.wikipedia.org/wiki/Lektine.

3 Wink M, et al: Handbuch der giftigen und psychoaktiven Pflanzen. Stuttgart, WVG, 2008.

4 Phytohaemagglutinin (kidney bean lectin); in Food and Drug Administration (ed): Bad Bug Book, ed 2. Foodborne Pathogenic Microorganisms and Natural Toxins Handbook. 2012.

www.fda.gov/downloads/Food/FoodborneIllnessContaminants/ UCM297627.pdf.

5 Frohe D: Viscum album L. Mistel; in Frohe D: Heilpflanzenlexikon, ed 8. Stuttgart, WVG, 2006.

6 6 Lei HY, Chang CP: Lectin of Concanavalin A as an anti-hepatoma therapeutic agent. J Biomed Sci 2009;16:10.

7 Swanson MD, et al: A lectin isolated from bananas is a potent inhibitor of HIV replication. J Biol Chem 2010;285:8646-8655.

$>8$ Khan F, et al: Lectins as markers for blood grouping. Med Sci Monit 2002;8:RA293-300.
Abb. 4. Blütenstand von Musa acuminata (Banane). Botanischer Garten Basel, 2012.

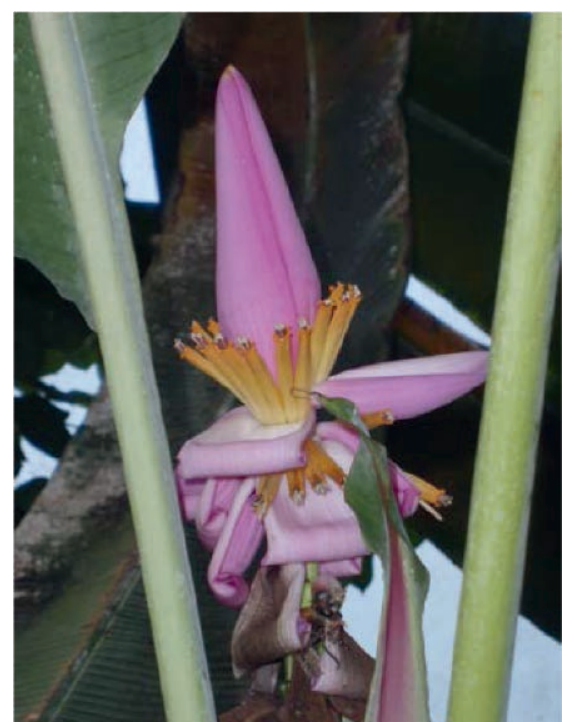

durch die Wahl eines bestimmten Lektins bewerkstelligt. Beispielsweise ist das Lektin von Phaseolus limensis (Lima-Bohne) spezifisch für Blutgruppe A, das Lektin von Griffonia simplicifolia (Afrikanische Schwarzbohne) spezifisch für Blutgruppe B und das Lektin von Sambucus nigra (Schwarzer Holunder) spezifisch für Blutgruppe $\mathrm{H}$ (0) [8]. 